\title{
Performance Optimization in Video Transmission over ZigBee using Particle Swarm Optimization
}

\author{
Iman Samizadeh, Hassan Kazemian, Ken Fisher and Karim Ouazzane \\ Intelligent Systems Research Centre, London Metropolitan University \\ 166-220 Holloway Road, London, N7 8DB, United Kingdom
}

\begin{abstract}
IEEE 802.15.4 - ZigBee is a wireless sensor targeted at applications that require low data rate, low power and inexpensive. IEEE 802.15.4 is limited to a throughput of $250 \mathrm{kbps}$ and is designed to provide highly efficient connectivity. Hence, IEEE 802.15.4 is not designed to transfer large amounts of data or MPEG-4 as its bandwidth is too low. In engineering and computer science often use optimization techniques, as do real environment applications in order to overcome complex issues and now this paper a solution has been accomplished by applying Particle Swarm Optimization (PSO) to improve the quality of transmitted MPEG-4 over IEEE 802.15.4. The proposed intelligent system should minimize data loss and distortion. The computer simulation results confirm that applying PSO in video transmission improve the quality of picture and reduce data loss when compared with the conventional MPEG video transmission in ZigBee.
\end{abstract}

Keywords: Index Terms_-Adaptive Quantization, MPEG-4, Particle Swarm Optimization, Rate Control, ZigBee, Video Transmission

\section{$1 \quad$ Introduction}

The IEEE 802.15.4 standard also known as ZigBee is a new frequency standard in wireless technology. It is designed to be cost-effective and is targeted at Low-Rate Wireless Personal Area Networks (LR-WPANs) with low power consumption, radio frequency applications [1], provides highly efficient connectivity and in many ways is similar to Wi-Fi and Bluetooth, operating in the same $2.4 \mathrm{GHz}$ Industrial Scientific Medical (ISM) band worldwide at a maximum data-rate of $250 \mathrm{kbps}, 868 \mathrm{MHz}$ band at a data rate of $20 \mathrm{kbps}$ in Europe and $914 \mathrm{MHz}$ band at $40 \mathrm{kbps}$ in the USA and Australia [2], [3].

IEEE 802.15.4/ZigBee's potential as a cost effective makes it highly likely that it will soon be used to transfer large amounts of data or stream videos. However, ZigBee bandwidth is very low for video transmissions over IEEE 802.15.4 networks and it would be difficult to achieve. Hence, in this paper a novel solution is presented to transmit digital video using the MPEG-4 compression technique over the ZigBee wireless sensor network with a sufficient quality.

adfa, p. 1, 2011.

(C) Springer-Verlag Berlin Heidelberg 2011 
MPEG-4 can be transported on existing transport layers and the majority of transport layers and network topologies are based on various forms of packet or cell switching technologies. Artificial Intelligence (AI) introduced to MPEG-4, and can decrease the amount of data that is transmitted, which can solve the problem and avoid congestion, whilst reducing the data transmission when a problem occurs during communication. Below is a brief history of the many different pertinent pieces of research that have been conducted in video streaming using AI and wireless communications.

Cheng and Chang came up with a method based on fuzzy logic in order to control congestion, whilst maintaining the quality of service [6]. They improved their model even further by introducing call admission control as well as congestion control on Asynchronous Transfer Mode (ATM) networks. In order to improve the performance of ATM networks, Huang et al., use a virtual leaky bucket with fuzzy logic control to manage the depletion rate in the bucket. Their simulation results show that the fuzzy leaky bucket system is effective in detecting source violation with low response time and the performance is significantly better than other mechanisms [8]. Likewise, Kazemian and Meng have introduced a solution, adding a fuzzy control system at the host controller interface [11]. This fuzzy control scheme was developed to transmit MPEG-4 over a Bluetooth wireless network to improve QoS in video streaming using a fuzzy approach [12].

PSO has also been applied in MPEG bit rate optimization. In the research conducted by Arachchi and Fernando, PSO-based bit rate optimization for MPEG-1/2 video coding has been studied and they have concluded that one of the significant problems in video compression schemes is the high fluctuation in the output data rate over the video sequence. These compression schemes, in general, utilize a rate control algorithm in order to maintain the output data rate at a constant level, regardless of the properties of the video sequence and the differences in the compression ratios of different picture types. Experimental results show that the proposed method can improve the average picture signal to noise ratio (PSNR) by more than $2 \mathrm{~dB}$ [13], [14]. According to the above research and the similarity between ZigBee, Bluetooth and other IEEE standards algorithms, and considering that MPEG-4 video files need a large bandwidth in order to stream over wireless networks, a novel solution would be to transmit MPEG-4 over IEEE 802.15.4 by introducing the PSO to the MPEG-4 applications. PSO is a relatively new AI technique and is a population-based stochastic optimization technique developed by Eberhart and Kennedy, inspired by the social behaviour of birds. The algorithm is very simple but powerful [15], [16]. PSO and the Genetic Algorithm (GA) represent population-based optimization heuristics for searching in high-dimensional spaces. The GA is an adaptive strategy and a global optimization technique [17]. PSO is very similar to the GA with much lower computational costs. The idea of PSO is that populations of potential solutions are intended to move collectively through a problem search space, under their respective algorithmic strategies, and ideally to a solution representing the global optimum [18]. PSO adapts behaviour and looks for the best solution vector in the search space. In PSO, a particle refers to a solution; each particle has a velocity that directs the "flying" of a 
particle as well as a cost value and fitness that is evaluated and minimized by the function. When there is a continuous optimization problem, each particle searches for a solution to it by flying through the search space following the optimum particles. The particle's flying experience and the experience of its neighbouring particles, decide the velocity of each particle. It is the local or global best that ultimately affects the behaviour of each particle to help it fly through hyperspace. Therefore, by observing the behaviour of the flock and memorizing their flying histories, all particles in the swarm can quickly converge to near-optimal geographical positions [19]. The idea proposed in this paper uses PSO, which requires a lot less computation, and accordingly it can be executed faster alongside MPEG-4 video compression. The proposed model has achieved an optimum level of quality of pictures whilst maintaining the ZigBee target bitrate, which has led to improvement in the quality of picture by reducing the data loss.

\section{PSO application to improve the quality of transmitted GOP in ZigBee}

The approach used to successfully transmit MPEG-4 videos over the ZigBee wireless network, which is aimed primarily at remote control and sensor applications, is the application of PSO to video compression whilst enhancing the QoS. In this paper, the problem-solving strategy decided on the use of PSO.Hassan et al., research results show the computational efficiency superiority of PSO over the GA. This is statistically proven with a $99 \%$ confidence level in seven out of the eight test problems investigated. Sivanandam and Deepa have compared PSO and GA for Lower Order System Modelling and their overall simulation results indicate that both Gas and PSO can be used in the search of parameters during system modelling. With respect to minimizing the objective function Integral Square Error, PSO determines a smaller value than GA. In terms of computational time, the PSO approach is faster than GA, although it is noted that neither algorithm takes what is considered as an unacceptably long time to determine the results [24]. Therefore, the proposed idea in this research uses PSO, which requires a lot less computation and accordingly it can therefore be executed faster.

The simulation presented in this paper explains the implementation of a transceiver for the ZigBee wireless communication system using Matlab, Simulink and MPEG-4 compression techniques, such as, motion compensated prediction, transform coding, quantization, entropy coding and other encoding processes. The proposed model mechanism is to reside in the IEEE 802.15.4 standard transmitter device side. The system is composed of an MPEG-4 encoder to input the data to a network that is organized into two nodes; transceivers and receivers, which is then passed onto a decoder. In addition, the simulation process is conducted by adding additive white Gaussian noise (AWGN) in a simulator of 802.15.4 device communication that comprises the transmitter radio channel. The input of the system is an AVI (interactive audio and video file) file for the encoder and the expected output file from the decoder is an AVI video file. The input file has the size of 176 pixels by 144 pixels and 
$64 \mathrm{kbit} / \mathrm{s}$ data rate. In this simulation, 240 frames or 20 Group of Pictures (GOP) for a period of 20 seconds are transmitted over the IEEE 802.15.4 standard network. The bandwidth in this simulation is the maximum value available in IEEE 802.15.4 ZigBee set at 250kbps. The GOP's range is given from 1 to 20 GOPs, which is approximately 240 frames. The other parameters are for the quantization; for Variable Bit Rate (VBR) the values are 8 for I-frames, 10 for P-frames and 25 for B-frames. For Constant Bit Rate (CBR) the parameters have been set to a constant value of 15 for all the frames I, P and B. MPEG-4 can work either in VBR or CBR to set the control parameters of a video encoder. Following the encoding, parameters are needed to control the output bitrate. The Quantizer Parameter (QP), or step size, is the most obvious parameter to vary, or rescale. This is because increasing the QP reduces the coded bitrate, whilst decreasing the QP will increase the coded bitrate. Quantization can be express as:

$$
Q O=\operatorname{round}(X / Q P)
$$

where $X$ is the input value, $Q P$ is quantizer size, which controls the range of the output $(Q O)$. The adaptive quantization in this simulation uses the initial value of 15 for each Q-set $(Q P)$ to start with, however, soon after running the simulation, it will override the value of its initial settings to decide adaptively on the required $Q P$ to find the best fit for the purpose and transitions rates.

In order to achieve the rate control and maintain the target bitrate or mean bitrate and to minimize, distortion in the decoded sequence, the Q-set has been modified during encoding. Using PSO, the output rate of the encoder, can be closely controlled during encoding and the optimum Q-scale size can be determined in an ad-hoc way. This approach should eliminate any data loss and packet drops. The target bit rate, is calculated based on the number of frames in the GOP and the minimum and maximum levels of bits that are available, which are known by calculating the prediction $\mathrm{P}$ frame rate. If the previous frame is an I-frame, it is used as a reference to predict the next frame's complexity and is allocated a suitable number of bits, following that the quantize step size for the following $\mathrm{P}$ and $\mathrm{B}$ frames is calculated. The desired bit rate or target rate is expressed in Equation 2:

$$
\text { Target rate }=\left(\frac{\text { Number of frames }}{\text { frame rate }(24)}\right) \text { ZigBee }
$$

Finding the bitrate of an uncompressed video, using resolution and frame rate and lossless video through approximations of quality, is expressed in Equation 3:

$$
\text { Bitrate }=(x \times \mathrm{y}) \times \frac{\mathrm{MF}}{\mathrm{B}} \times \text { Rate }
$$

where $x$ is the frame width, with the value of the video file as 176 pixels, and $y$ is the height with the value of 144 pixels. The number 4 is the value of MF (Motion Factor), which is divided by 8 bits, and therefore, 1000 is the value of frame bitrate. The value 
of bitrate then is passed to the PSO for optimization. In PSO, particles move over a specified D-dimensional search space at different random or heuristically velocities and positions. The algorithm updates the velocity and position of each particle in the swarm by learning from its neighboring particles. Its own fitness is then evaluated and a good experience is reached. The basic particle swarm model can be explained in Equation 6 [26], [27]. In a $D$-dimensional search space, the position vector of the $i$-th particle is given by $X_{i}=\left(x_{i, 1}, x_{i, 2}, x_{i, D}\right)$ and the velocity of the $i$-th particle is given by $V_{i}=\left(v_{i, 1}, v_{i, 2}, v_{i, D}\right)$. Positions and velocities are adjusted and the objective function is to optimize i.e. $f\left(x_{i}\right)$ is evaluated with the new positional coordinates at each time-step. The velocity and position update equations for the $d$-th dimension of the $i$ th particle in the swarm may be represented as explained in Equation 4 [26], [27]:

$$
\begin{gathered}
v_{i, d, t}=w \times v_{i, d, t-1}+C_{1} \times \operatorname{rand}_{1} \times\left(p_{i, d, t-1}^{t}-x_{i, d, t-1}\right)+C_{2} \\
\times \operatorname{rand}_{2} \times\left(p_{i, d, t-1}^{g}-x_{i, d, t-1}\right) \\
x_{i, d, t}=x_{i, d, t-1}+v_{i, d, t}
\end{gathered}
$$

where $\operatorname{rand}_{1}$ and $\operatorname{rand}_{2}$ are random positive numbers uniformly distributed in $(0,1)$ and are drawn anew for each dimension of each particle.

$P_{i}^{l}$ is the personal best solution found so far by an individual particle while $P_{i}^{g}, i$ represents the best particle in a neighborhood of the its particle for the lbest PSO model. Note that in PSO, a neighborhood is defined for each individual particle as the subset of particles, which it is able to communicate with. The gbest for PSO may be regarded as a special case of the lbest model where the entire swarm acts as the neighborhood of any particle and $P_{i}^{g}, i$ simply becomes the globally best position found so far by all the particles in the population. In lbest PSO, if at any iteration a particle is the best in its neighborhood, then the velocity update formula as presented in Equation 5 for this particle will be [27], [28]:

$$
v_{i, d, t}=w \times v_{i, d, t-1}+C_{1} \times \operatorname{rand}_{1} \times\left(P_{i, d}^{I}-x_{i, d, t-1}\right)
$$

The variables in the PSO system of equations are summarized in Table $\mathbf{1 .}$ 


\begin{tabular}{|c|l|}
\hline$V_{i}$ & The particle velocity. \\
\hline$X_{i}$ & The particle position (test solution). \\
\hline$t$ & Time \\
\hline$P_{i}^{l}$ & $\begin{array}{l}\text { The particle's position (previous) that resulted in the best fitness } \\
\text { so far. }\end{array}$ \\
\hline$P_{i}^{g}$ & The neighborhood position that resulted in the best fitness so far. \\
\hline$d$ & $D$-dimensional search space \\
\hline
\end{tabular}

Table 1. The PSO Variables

The lower and upper boundaries of the Q-scales are 1 and 31 respectively. The lower and upper boundaries constitute the search-space. The boundaries force the optimization method to move the candidate frame back to the boundary value if it has exceeded the boundaries that are denoted as $\vec{b}_{I o}$ and $\vec{b}_{u p}$ up as formulated in Equation 6 [29]:

$$
f:\left[\vec{b}_{\text {Io }}, \vec{b}_{\text {up }}\right] \rightarrow \mathbb{R}
$$

If optimization problems are $f$ functions these are explained in Equation 7 of the following form [30]:

$$
f: \mathbb{R}^{n} \rightarrow \mathbb{R}
$$

Assuming that $f$ is a minimization problem, meaning that it is searching for the candidate solution $\vec{x} \in R^{n}$ with the smallest value $f(\vec{x})$ using the following example: Find $\vec{x}$ such that $\forall \vec{y} \in \mathbb{R}^{n}: f(\vec{x}) \leq f(\vec{y})$

It is often not possible to find the exact optimum and a candidate solution of sufficiently good quality must be used instead [28], [29]. The evaluation of frame rates is then passed into the Rosenbrock function [30]. The first input argument is the frame rates to be evaluated. Instead of iteratively recalculating the number of particles from the dimensionality of the position matrix, the information is passed to the function through the second input argument, which corresponds to each frame row or rate that has been evaluated [31]. Personal and global bests, including the best Fitness, are updated based on how well they minimize the following Equation 8 [30], [31].

$$
\begin{aligned}
& f(\vec{x})=\sum_{i=-30}^{30} \sum_{j=j-1}^{n-1}\left(100\left(x_{j+1}-x_{1}^{2}\right)^{2}+\left(1-x_{i}\right)^{2}\right) \\
& -30 \leq x_{i} \leq 30
\end{aligned}
$$

The first input argument is the frame rates to be evaluated in Rosenbrock function. Instead of iteratively recalculating the number of particles from the dimensionality of the position matrix, the information is passed onto the function through the second input argument, which corresponds to each frame row or rate that has been evaluated. Personal and global bests, including the best fitness, are updated based on how well 
they minimize [31], [32]. After training the data the result of the PSO then determines the Q-Step size for each GOP.

\section{Computer simulation results}

PSNR (Peak Signal-to-Noise Ratio) is a way of telling approximately the human perception of the reconstructed quality. For this reason, a reconstruction may sometimes appear to be closer to the original than others do. PSNR is formulated in Equation 7 [11]:

$$
P S N R=10 \log \frac{\left(2^{n}-1\right)^{2}}{M S E}=10 \log \frac{255^{2}}{M S E}
$$

A random frame sample taken from the most fluctuated range of data. Error! Reference source not found. is the sample that has been taken for this evaluation. It is comparing the PSNR of VBR, CBR and PSO methods. The result shows that the PSNR result or the sample frame in VBR after transmission is $20.9351 \mathrm{~dB}$.

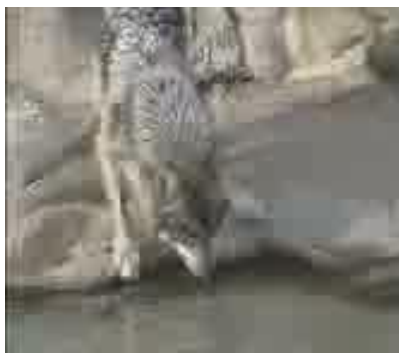

Fig. 13. Sample frame transmitted in VBR

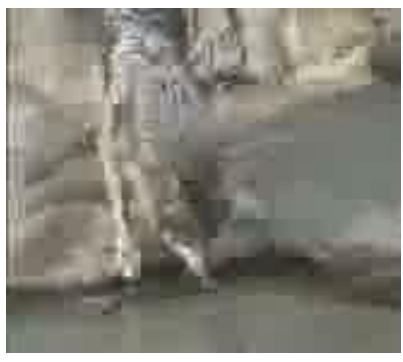

Fig. 14. Sample frame transmitted in CBR

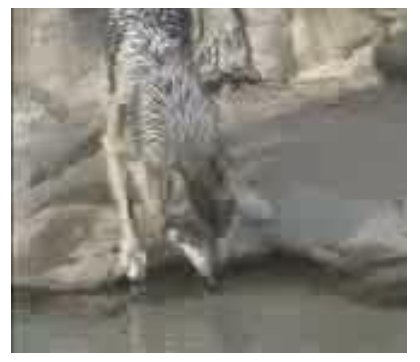

Fig. 15. Sample frame transmitted in PSO

The PSNR result compared to the source in CBR using the same frame number is shown in Error! Reference source not found. and is $17.83 \mathrm{~dB}$ The PSNR result on the source of adaptive quantization using PSO on the same frame number is shown in Error! Reference source not found.. In this test, PSO conducts the optimum result in between VBR and CBR with value of $20.88 \mathrm{~dB}$; that is $4.37 \%$ less than VBR. Even though a small percentage of quality of the video object is lost, the PSO method compensates for this by remaining within the bandwidth target rate limit of IEEE 802.15. Like with the VBR, PSO has a greater value than CBR. The frame rate of the sample frame is compared to evaluate the PSNR to transmission rate and the encoding quality. In VBR it is $398 \mathrm{kbps}$, in CBR the frame rate is $279 \mathrm{kbps}$ and in PSO it is $237 \mathrm{kbps}$.

The results in Table 2 show that VBR has a better quality. However, because of large frame rate size the frame is not suitable for the given rate of $250 \mathrm{kbps}$ in ZigBee. CBR, with the constant value of Q-step, also has a large frame rate but it has very low quality. Therefore, the results prove that with use of PSO and introducing an adaptive 
quantization it can achieve a good balance in higher PSNR than other commonly used methods, whilst improving the quality of the image during the encoding, and adaptively managing the best frame rate at the target bitrate.

\begin{tabular}{|l|l|l|}
\hline Method & Frame rate & PSNR \\
\hline VBR with Gaussian & $379 \mathrm{kbps}$ & $20.93 \mathrm{~dB}$ \\
\hline CBR with Gaussian & $266 \mathrm{kbps}$ & $17.83 \mathrm{~dB}$ \\
\hline PSO with Gaussian & $209 \mathrm{kbps}$ & $20.88 \mathrm{~dB}$ \\
\hline
\end{tabular}

Table 2. PSNR and frame rates transmitted with white Gaussian noise

The PSNR results for the PSO algorithm present the minimum value of $17.83 \mathrm{~dB}$ and a maximum value of $20.93 \mathrm{~dB}$. To compare the different PSNR results for each method, the PSNR results are grouped together and presented in Fig.1.

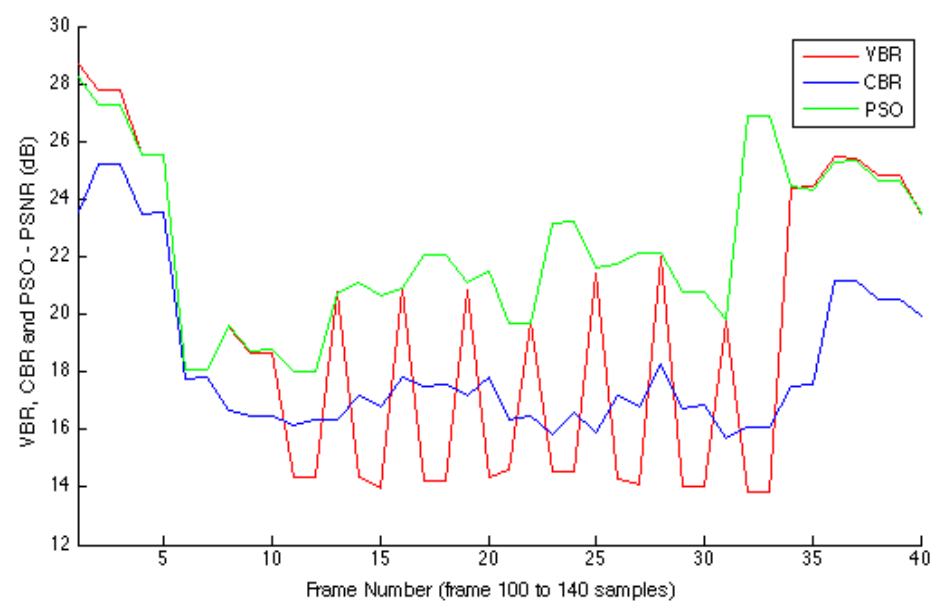

Fig. 1. PSNR in VBR, CBR and PSO

The minimum and maximum numbers for each method is listed in Table $\mathbf{3}$.

\begin{tabular}{|l|l|l|}
\hline \multirow{2}{*}{ Method } & \multicolumn{2}{|l|}{ PSNR } \\
\cline { 2 - 3 } & $\operatorname{Min}(\mathrm{dB})$ & $\operatorname{Max}(\mathrm{dB})$ \\
\hline VBR & 13.84 & 28.73 \\
\hline CBR & 15.72 & 25.18 \\
\hline PSO & 17.91 & 28.30 \\
\hline
\end{tabular}

Table 3. Minimum and Maximum PSNRs

The PSNR shows that the PSO method carried the highest value in the minimum group at a value as low as $17.91 \mathrm{~dB}$. Whilst having an optimum value of 28.30 within 
the maximum group, which is less than the Maximum PSNR value of the VBR method and greater than the PSNR maximum value of the CBR.

\section{Conclusion}

The PSNR shows that the proposed idea carries an optimum value of PSNR, which is less than the maximum PSNR value of the VBR method and greater than the maximum PSNR value of the CBR method. These results determine that the proposed method is superior to both the VBR and CBR methods and the use of PSO can improve MPEG-4 streaming by using an optimum level of the available bandwidth. The proposed model can increase the efficiency of the bandwidth, prevents data loss and most importantly it improves QoS and enables MPEG-4 video to be transmitted over IEEE 802.15.4 standard. Also, computer simulation results confirm that the use of PSO as an optimization model to develop an adaptive scalar quantization video coding, improves the quality of picture whilst reducing data loss and communication delay, when compared with conventional MPEG-4 video transmissions.

\section{$5 \quad$ References}

1. IEEE Computer Society, (August 31, 2007). IEEE Standard 802.15.4a-2007, (2007)

2. IEEE Std 802.11b-1999 (R2003) - the IEEE 802.11b specification. e-book. Available at: http://standards.ieee.org/getieee802/download/802.11b-1999.pdf [Online] Accessed 17 Oct 2009, (2003)

3. Farahani, S.: ZigBee Wireless Networks and Transceivers - ISBN: 978-0-7506-8393, (2008)

4. ATM Forum, The User Network Interface (UNI), v. 3.1, ISBN 0-13-393828-X, Prentice Hall PTR, 1995, pp. 2, (1995)

5. Haskell, P., Messerschmitt, D.: Resynchronization of motion compensated video affected by ATM, cell loss, in ICASSP-92, San Francisco, CA, USA, Mar. 1992, vol.3, pp. 545-548, (1992)

6. Chang, C., Cheng, R.: Traffic control in an ATM network using fuzzy set theory. In Proceedings of the IEEE INFOCOM'94 Conference, Toronto, Canada, June 1994. IEEE Communications Society. pp. 1200--1207 (1994)

7. Ascia, G., Catania, V., Ficili, G., Palazzo, S., Panno, D., VLSI, A.: fuzzy expert system for real-time traffic control in ATM networks. IEEE Transactions on Fuzzy Systems, volume 5 issue 1, February 1997, pp. 20--31, (1997)

8. Cheng, R.G., Chang, C.J.: Design of a fuzzy traffic controller for ATM networks. IEEE/ACM Transactions on Networking, volume 4 issue 3, June 1996. pp. 460-- 469, (1996)

9. Huang, Ming-Chang., Seyed Hossein Hosseini, Vairavan, K., Hui Lan.: Fuzzy Congestion Control and Policing in ATM Networks. International Journal of Engineering (IJE), Volume 3, Issue 1, pp. 65--84, (2009)

10. Wua, Shih-Lin., Fan-Jianga, Sui., Choub, Zi-Tsan.: An efficient quality-of-service MAC protocol for infrastructure WLANs, Journal of Network and Computer Applications 29 pp. 235-261, (2006) 
11. Kazemian, H., Meng, L.: A fuzzy control scheme for video transmission in Bluetooth wireless. INS - Information Sciences, volume 176 issue 9, May 2006, pp. 1266--1289, (2006)

12. Kazemian, H., Meng, L.: An adaptive control for video transmission over Bluetooth. IEEE Transactions on Fuzzy Systems, volume 14 issue 2, April 2006, pp. 263--274, (2006)

13. Emilio, C.G., Willea, Melliab, M.: Selected Papers from the 3rd International Workshop on QoS in Multi service IP Networks (QoS-IP 2005) Algorithms for IP network design with end-to-end QoSnext term constraints star, open. Computer Networks, Volume 50, Issue 8, 6 June 2006, pp.1086--1103, (2006).

14. Arachchi, H.K., Fernando, W.A.C.: PSO based bit rate optimization for MPEG-1/2 video coding. Image Processing, 2005. ICIP 2005. IEEE International Conference on 11-14 Sept. 2005, Volume: 2, pp.- 329--32, (2005)

15. Kennedy, J., Eberhart, R. C., Shi, Y.: Swarm Intelligencell, Morgan Kaufmann Publishers, San Francisco, CA, (2004)

16. Kennedy, J., Eberhart, R. C.: Particle swarm optimizationl, Proc. IEEE Int'l Conf. on Neural Networks, IV, 1942-1948. Piscataway, NJ: IEEE Service Center, (1995)

17. Brownlee, Jason.: Clever Algorithms - Nature-Inspired Programming Recipes - ISBN: 978-1-4467-8506-5, (2011)

18. Owen, A., Harvey, I.: Adapting Particle Swarm Optimization for Fitness Landscapes with Neutrality, 1-5 April 2007, IEEE Swarm Intelligence Symposium, pp. 258--265 (2007)

19. Chunlin, Ji., Zhang, Y., Tong, M., Yang, S.: Particle Filter with Swarm Move for Optimization - Proceedings of the 10th international conference on Parallel Problem Solving from Nature: PPSN X - pp. 909--918. (2008)

20. Zainaldin, A., Lambadaris, I., Nandy, B.: Video over Wireless Zigbee Networks: MultiChannel Multi-Radio Approach. Wireless Communications and Mobile Computing Conference. IWCMC '08. International. 6-8 Aug. 2008, pp. 882-- 887 (2008)

21. Shilpa, K.S., Narayan, D.G., Kotabagi, S., Uma, M.: Suitability Analysis of IEEE 802.15.4 Networks for Video Surveillance. Computational Intelligence and Communication Networks (CICN), International Conference on. 7-9 Oct. 2011 - pp. 702--706 (2011)

22. Hassan, R., Cohanim, B., Weck, O. D., Venter, G.: A Comparison Of Particle Swarm Optimization And The Genetic Algorithm. Structures, Structural Dynamics, and Materials Conference 2005, pp. 1-13, (2005)

23. Azarkish, H., Farahat, S., Masoud, S., Sarvari, H.: Comparing the Performance of the Particle Swarm Optimization and the Genetic Algorithm on the Geometry Design of Longitudinal Fin. World Academy of Science, Engineering and Technology. vol. 1, issue 1, February 2012, pp. 1--5, (2012)

24. Sivanandam, S. N., Deepa, S., N.: A Comparative Study Using Genetic Algorithm and Particle Swarm Optimization for Lower Order System Modelling. International Journal of the Computer the Internet and Management. Date 2002; pp. 1--10, (2002)

25. Richardson, I. E. G.: H.264 and MPEG-4 Video Compression Video Coding for NextGeneration Multimedia: Video Coding for Next-generation Multimedia - ISBN-10: 0470848375 (2003)

26. A. Abraham, S. Das, S. Roy, Swarm Intelligence Algorithms for Data Clustering, Soft Computing for Knowledge Discovery and Data Mining 2008, Springer, ISBN 978-0-38769934-9, pp. 279-313, (2008)

27. S. Ghosh, S. Das, D. Kundu, K. Suresh, A. A. Genev, Inter-particle communication and search-dynamics of lbest particle swarm optimizers: An analysis. Nature-Inspired Collective Intelligence in theory and Practice, Vol. 182, Issue 1, 1 January 2012, pp. 156--168, (2012) 
28. Y. Penga, B. L. Lua, A hierarchical particle swarm optimizer with latin sampling based memetic algorithm for numerical optimization, Applied Soft Computing Journal, Elsevier, Jun 20912, pp 1-14, (2012)

29. Pedersen, M.E.H.: SwarmOps for Matlab - Numeric \& Heuristic Optimization, November 2010, http://www.hvass-labs.org/projects/swarmops/matlab/ (2010)

30. Pedersen, M.E.H.: SwarmOps for Matlab - Numeric \& Heuristic Optimization, November 2010, http://www.hvass-labs.org/projects/swarmops/matlab/, (2010)

31. H. H. Rosenbrock, An Automatic Method for Finding the Greatest or Least Value of a Function, Computer Journal. 3, 1960, pp. 175-184, (1960)

32. J. J. Moré, S. B. Garbow, E. Kenneth, Hillstrom, Algorithm 566: FORTRAN Subroutines for Testing Unconstrained Optimization Software [C5], [E4]. Journal ACM Transactions on Mathematical Software, Vol 7, Issue 1, March 1981, pp. 136-140, (1981) 